\title{
Engineering roles and identities in the scientific community: toward participatory justice ${ }^{1}$
}

\author{
V. Pronskikh ${ }^{2}$ \\ Fermi National Accelerator Laboratory, Batavia, IL 60510-5011, USA ${ }^{3}$
}

\begin{abstract}
This paper seeks to examine the roles and identities of engineers constituting one of the fundamental, but a completely indescribable community in modern big science with particle accelerators. Large communities of accelerator and detector specialists, which replaced experimenters and instrumentalists of the middle of the last century, themselves exhibit a complex structure and are divided. However, this division is in turn grounded on the division of those whose activities focus on the phenomena of nature considered independent of human beings and those who design processes and phenomena of an artificial, technical nature. Nevertheless, in terms of their modus operandi and identity, the kinship between engineers and experimental scientists is considerable. I argue that such exclusion of the engineering community from epistemic practices can serve as an example of participatory injustice. As one of the ways to transcend participatory injustice, I suggest that the communities should be encouraged to work together in epistemically tantamount roles while structural hindrances to the mobility between communities need to be alleviated.
\end{abstract}

Keywords: engineering, high-energy physics, communities, roles, mobility, participatory justice

\section{Introduction}

Contemporary fundamental science has a number of significant contrasts with the science of the early 20th century. Having become a complex social institution in its very structure, it demanded the eliciting and scrutinizing of the communities that make up research teams and the features of their interaction. Several outstanding studies have been undertaken by a number of historians, philosophers, and sociologists of science (Galison 1987; Pickering 1988; Collins 2002, Hoddeson et al. 2008, Knorr-Cetina 1999, Traweek 1988, Latour and Woolgar 1979). The objective of this work is to examine the phenomenon of the big science community in its development and the influence of the rise of an elementary particle accelerator and a complex elementary particle detector in it on the amplification of the social structure, the deepening of the epistemic division

\footnotetext{
${ }^{1}$ Talk accepted for presentation at PSA2020: The 27th Biennial Meeting of the Philosophy of Science Association (Baltimore, MD; 18-22 Nov 2020).

2 vpronskikh@gmail.com

${ }^{3}$ Fermi National Accelerator Laboratory is operated by the Fermi Research Alliance, LLC under Contract No. DEAC02-07CH11359 with the U.S. Department of Energy, Office of Science, Office of High Energy Physics.
} 
of labor and the need for lengthy engineering activity at the stage of preparation of experiments. To accomplish that task, it becomes necessary to clarify the roles of members of scientific communities and the dynamics of changes in their structure, to discuss the difficulties of classification and identities of community members, as well as issues that arise at the present stage in the relationship of scientific and engineering activities and possible ways to resolve them.

Notably, the rise of the accelerator in the 1930s-1940s was the first milestone in the development of a modern complex physics experiment and the complication of the structure of communities associated with large-scale experiments in high-energy physics. First, the emergence of such a large and complex device as an accelerator led to the appearance of accelerator physicists (and engineers) as scientific and technical specializations. Their task comprised the design of the accelerator, the calculation and optimization of its parameters, as well as ensuring its operation (providing particle beams accelerated to the required energies and intensities, to the community of experimentalists). The creation of the accelerator not only led to the spatial separation of the theoretician working silently in their Ivory Tower from the experimentalist, who now had to spend most of their time in the experimental halls near the accelerator, where their installation was established. In addition to the communities of experimentalists and theorists, with the beginning of experiments on accelerators, a community of accelerator specialists emerged, engaged in the creation and maintenance of the accelerator machine. Galison (1987) also introduced a community of instrumentalists involved in the creation of scientific instruments and installations, and formally accelerator scientists could be classified as such a community because the accelerator is a technically sophisticated device whose operating principles are essentially based on classical electrodynamics. However, in such a case, it would turn out to be very heterogeneous because the expertise of the accelerator researcher and the instrumentalist, who builds, for example, a particle detector, will differ.

Beginning from the 1970s and finally by the beginning of the 1990s, a detector-a device in which particles born in collisions of a beam are detected, and their characteristics are identified by measurement, ended up to be so tangled and universalized that it morphed into the central object of the experiment that in many respects began to designate the long-term directions of research in accelerator laboratories (Hoddeson 2008). The structure of the corresponding communities began to change accordingly. Now, experimenters became engaged in detector calculations and design for a long time, taking on some of the tasks that were previously assigned to instrumentalists (in particular, engineers), then undertaking measurements with it and analyzing the data for an equally long time. After a series of measurements, they often continued to improve the design of the detectors and their components, returning to the engineering kind of work, again measuring and analyzing until the range of tasks that can be solved with this type of detector and accelerator capabilities was exhausted. All together, it took dozens of years, sometimes the whole conscious life of the experimentalist of this particular type of detector or the same detector. However, the communities of accelerator and detector researchers themselves are also heterogeneous. We shall consider their structure in more detail.

\section{Accelerator and detector researchers}

Starting from the 1990s, one can assume that instead of communities of theorists, experimenters, and instrumentalists, in high-energy physics, one should talk about communities of theorists, 
accelerator, and detector researchers. The structure of the theoretician community has not changed much, while other communities have undergone the greatest changes since the first third of the 20th century. The community of accelerator specialists now builds and maintains the accelerator, and the community of detector specialists - the detector. Instrumentalists can now be considered a subset of detector and accelerator specialists, and the dividing line between them and detector experimental experts is rather blurred. At the stage of creating the setup, the distinction between experimental scientists and nonscientists is such that, although they both study processes in the detector on computational models, the former focus more on aspects related to future searches for a useful signal, its reconstruction (reconstruction of events occurring in the detector by triggering numerous sensors), while the second - on aspects related to ensuring the overall operability of the installation. The second difference between them becomes evident after the beginning of measurements at the setup, when the scientists participate in the data acquisition, and then enter on processing and analysis of the data, while the instrumentalists set about the creation of other installations and instruments.

Until about the 30s of the 20th century, the role of an instrumentalist did not exist because their functions were divided between the experimentalist and engineer as follows: the experimentalist formulated the technical requirements for the device (installation) to the engineer (industry) in the form of a set of requirements and those independently manufactured the device, most of which was standard and serial. Then, the experimentalist performed measurements on the setup, performing its adjustment as necessary, as well as data analysis, which was quite simple and not requiring separate education and specialization. With the birth of Big Science and the resulting complexity and uniqueness of the installations, the technical design specifications are becoming a joint product of experimentalists and toolmakers, resulting from a compromise and trade-off between a multitude of installation requirements. In this sense, the instrumentalist (and nowadays, the detector and accelerator researchers) is a transitional type between the engineer and the experimenter, and their own instrumentalists appear in both the accelerator and detector communities.

\section{Roles and specializations in megascience}

Each of these communities now become heterogeneous (see Table 1). Accelerator specialists also began to be divided into theorists (calculators) performing computational modeling of the particle acceleration, experimentalists who conducted experimental measurements of the developed accelerator assemblies to help create its technical theories, as well as engineers manufacturing the accelerator assemblies and performing their tuning and adjustment. Detector scientists are divided by the type of detector unit, which they simulate and build, and then support during the measurements and data which they own for analysis after the experiments. With the advent of the era of complex hybrid detectors, the detector began to consist of several complex, but heterogeneous system units, for example, such as a time-of-flight system, calorimeter, tracker, or shielding against the cosmic background. Each of these units, from its design period until the completion of the experiment, was under the responsibility of a certain group of experimentalists, which assumed both a number of technical issues and a physical interpretation of the data harvested from it. These groups together form a community of detector scientists. Engineers are entrusted with the development of installations, accelerators, and their units according to the technical specifications, maintenance of installations, accelerators, and software. 


\begin{tabular}{|c|c|c|}
\hline Area & Role & Specialization \\
\hline \multirow[t]{3}{*}{ Accelerator } & \multirow[t]{2}{*}{ Accelerator Physicist } & Accelerator Theorist \\
\hline & & Accelerator experimentalist \\
\hline & Engineer & Accelerator Engineer \\
\hline \multirow[t]{4}{*}{ Detector } & \multirow[t]{3}{*}{ Detector physicist } & Unit scientist \\
\hline & & Data measurer \\
\hline & & Data analyst \\
\hline & Engineer & Unit engineer \\
\hline \multirow[t]{2}{*}{ Phenomena theory } & \multirow[t]{2}{*}{ Theorist } & Model developer \\
\hline & & $\begin{array}{l}\text { Developer of calculation tools } \\
\text { and methods }\end{array}$ \\
\hline \multirow[t]{3}{*}{ Computing } & \multirow[t]{2}{*}{ Programmer } & \\
\hline & & System Tools Developer \\
\hline & Engineer & Software Maintenance \\
\hline
\end{tabular}

Table 1. The structure and functions of communities in high-energy physics.

\section{Experimentalists' identity and engineering}

To grasp the social processes in the high-energy physics laboratory, the nature of community interactions, their similarities, and dissimilarities, it is necessary to elucidate the identities of their members. As one of the signs of the experimenter's "identity shift," characteristic of the period of the 1970s, the awkwardness felt by the experimental physicist toward others (including the engineer) in the laboratory began to be noted (Galison 1997, 5). This "identity shift," was ascribed to the fact that the very nature of experimentation has changed: if earlier the experimenter's work was unambiguously associated with the design of the installation, the development of experimental procedures, the application of these procedures, the recording of results, and their theoretical analysis, then later the experimenter was considered to be the one who only analyzes the data harvest hiding behind the monitor a long distance away from the installation threshing mill. Hence, it became impossible to have a single view of what can be considered experimentation (which is reflected in Table 1). Another distinctive feature of this stage of the development of science can be considered the complex contradiction between the experimentalist and engineer on the one hand, and the productive tension between the experimentalist and theorist on the other (Galison 1997, 5).

For the sake of our analysis, in the above claims, we highlight the following central narratives: 1) the contradictions between the experimenter and other specialists of the scientific laboratory (theorist and engineer) that have been growing since the 1970s and 2) the emergence of experimentalists who were not engaged in the activities previously considered traditional, such as 
creating a facility and experimental procedures for it. These observations appear to be based on the following premises.

First, the community of experimenters implicitly related to detector specialists (as reflected in Table 1) is not uniform but covers a wide range of activities. As was noted, experimentation begins to shift toward data analysis. In practice, it is often believed that, because data analysis constitutes an interpretation of the processes occurring in the detector, in terms of high-level theories, bearing upon the language of instrumental theories in which the principles of the functioning of detectors are rooted, the outcome of this procedure is what directly becomes the experimental result. Because obtaining the measurement result is the experiment's main aim, therefore, the experimentalist, first of all, can be deemed the one whose activity immediately delivers the result, that is, an analysis of detector data.

Notably, most of the participants in the analysis of data (experimentalists) in the period preceding the acquisition and analysis of data are also engaged in the creation of detectors and procedures for them. Thus, the scope of their activity also partially covers that which belongs to the expertise of instrumentalists (who may also be engineers) - the creation of instruments. On the one hand, considering the fact that the creation of the device and the corresponding procedures take a long time (years and even tens of years), the experimenter had to devote a lot of time to the activities that are very close to engineering ones, de facto becoming a highly professional engineer. This may raise a legitimate question, why is one of them identified as a detector physicist (experimentalist) and the other as an engineer when their work and professional expertise are so similar? On the other hand, the epistemic distinctions in the nature of their work also blurred: the experimental search, in addition to being carried out, as before, in terms of the dominant theories, was increasingly guided and determined by these theories; the discovery of new phenomena was increasingly dependent on the development of high-level theories. Thus, experimentation became more and more tangibly the construction of theoretical natural objects, which was closer to the work of the designer than before. This became especially pronounced during the period of success of the Standard Model in elementary particle physics, which predicted many particles that were subsequently measured experimentally. This could not but affect the identity of the experimentalist, as well as the perception of the experimenter by the engineer as a theoretician.

The shift of identity, therefore, arose in connection with the need for lengthy engineering work for the experimentalist (because the creation of the installations took a long time and was not serial due to their uniqueness), on the one hand, and the increased constructiveness of the experimentation itself, on the other. This entailed the actual blurring of the lines between the nature of the work of the experimentalist and engineer, which was initially opposed much due to distinct educational trajectories, which are also linked by the mass consciousness with the level of possible scientific horizons and achievable professional competencies.

Why is a scientist more prestigious than an engineer?

Joseph Martin (Martin 2017) has recently argued that prestige of different social and epistemic groups in science, for example, particle physics and applied science (or engineering), is asymmetric (the latter being the least prestigious). The question of what constitutes an engineer's identity requires, first and foremost, an answer to two interrelated questions: Who is an engineer and what features make a person an engineer? Modern literature on identity theory distinguishes cognitive (internal) and social (external) identity (Anderson 2010; Wenger 1998). In the case of an engineer, the former part is predicated upon what they know about the profession, how they 
understands their role, what they want (may want) or do not want (may not want) to know professionally. This part is set not only by the engineer's personal cognitive peculiarities but also by the interrelation of their professional role as an engineer with the whole variety of other roles their play in life. The latter part, the social one, is not the traits that an engineer acquires in the course of professional practice, but those shaped by their membership in a social group. The latter part is formed by the community as a result of belonging to it, as well as by other communities and society as a whole, with which the engineer interacts and in which the attitude toward engineers as a social group is defined. This view raises the question of the stability of such an identity and the need for such conditions in the formation of the so-called engineering identity in general. Speaking of engineers as a social group, it becomes necessary to distinguish between an engineer, as the holder of engineering education, and an engineer as a performer of the role of an engineer.

To establish what constitutes an engineer's identity in science, one has to answer the initial question of what features and functions make one an engineer. In the practice of scientific laboratories in basic science (for example, elementary particle physics), the role of an engineer implies working with complex technical systems, but first, we will clarify what the disparities between engineers and scientists boil down to. Historically, these distinctions are rooted in an understanding of the very nature of the activities of these communities and the goals for which these activities, namely scientific and technical research, are oriented. Most approaches to distinguishing science and technology in one way or another reflect the Aristotelian distinction between $\dot{\varepsilon} \pi \imath \tau \eta \dot{\mu} \eta$ (episteme) as knowledge, understanding, or cognition and $\tau \dot{\varepsilon} \chi \nu \eta$ (techne) as craft or practical art. The first, according to Aristotle, is a theoretical knowledge of eternal and universal things that exist by virtue of their necessity; the second is the creation of transient and perishable, i.e., practical things. From here originates the ontological distinction between "knowledge of what" and "knowledge of how," knowledge of the true (first) and useful (second). In this regard, a "dichotomy of intellectual status" arose in science and society (Boon 2011, p. 63): higher status of science and lower of technology. At the same time, several authors point out the difficulties of discerning scientific and technical knowledge in modern science, and also advocate the possibility of considering them as either including one another (technical includes epistemic), or even as independent of each other (Boon 2011).

Nonanalyzing data detector and accelerator researchers can be classified as technically oriented scientists (except when they are studying new phenomena during the development of instruments). Engineers (they are not included in the classification (Galison 1987) because they are not classified as scientists) are not engaged in the science of independent development of new devices or study of new phenomena in technical systems, but operate and establish such systems or develop in accordance with the terms of reference, which are formulated by scientists. An engineer who is developing a new system or exploring it according to our view should be classified as a scientist. Thus, the physics of high energies retains the basis of the Aristotelian dichotomy of epistemic and technical which is reflected in the hierarchy of activities and communities in science from pure theorizing about natural phenomena (epistemics) down to technology applications (engineers). Signs of dichotomy remain, however, because even in mixed, intermediate cases, such as those of detector experimental physicists, their activities are clearly divided into two types of roles: the design of the device (for example, the tracker) is technical, its operation is also technical, and the analysis of data with the formulation of theoretical statements is scientific (epistemic). The same applies to the detector scientist, in whose work the epistemic part (the study of new natural phenomena suitable for creating new devices) and the technical (construction, design, and 
operation of devices) can clearly be traced. At the poles are theorists, all of whose roles are epistemic and engineers, all of which are technical (see Figure 1).

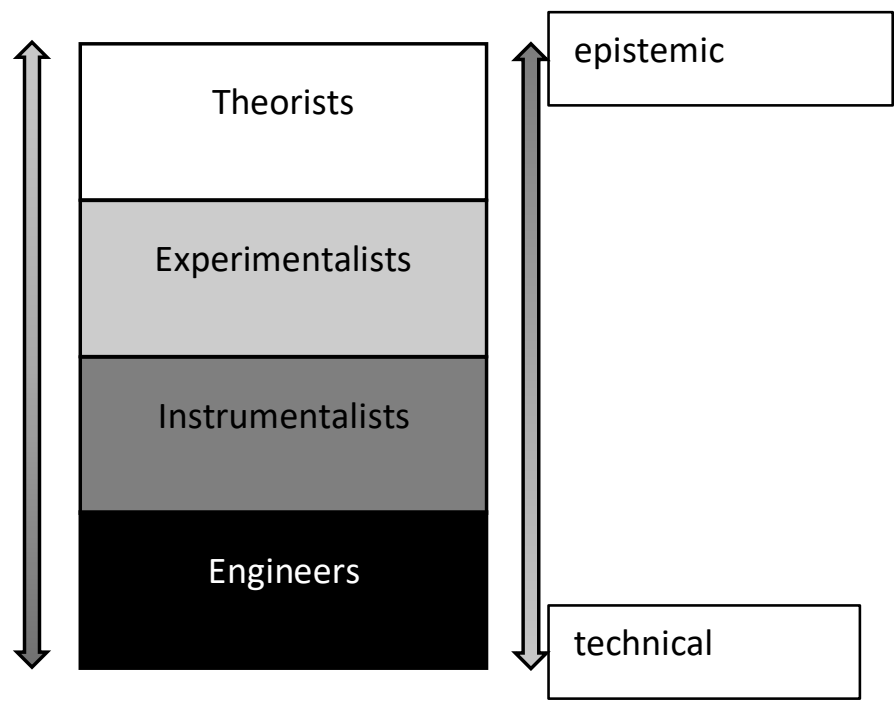

Figure 1. Epistemic hierarchy of communities in high-energy physics. Detector, as well as accelerator, researchers perform both epistemic and technical roles (which clearly differ, preserving the dichotomy), however, if the technical roles of the experimentalist (data analysis) are subjected to the measurement of the phenomena under scrutiny as an immediate goal, then even the epistemic goals of instrumentalists are resigned to improving the device designs (for example, of a particle accelerator), in connection with which the experimenters find themselves higher in the epistemic hierarchy. Here we assume the data-analyzing detector researchers to be experimentalists while both nondata-analyzing detector scientists and accelerator researchers are instrumentalists.

\section{Engineering and epistemic justice in megascience}

Thus, we have argued that the dissimilarity between engineering and other types of scientific activity do not resolve into the identity or constructive nature of the activity, but, first of all, are governed by perceived property relations and the rights claimed to experimental data that arise due to involvement in the data acquisition process. It is immixture in epistemically significant practices and affiliation in the data-harvesting community that come to the forefront in distinguishing an experimentalist from an engineer. Restrictions on the access of certain groups to epistemic practices based on their social or professional group membership and external identity raise the issue of epistemic justice.

Although epistemic injustice has been extensively discussed in the philosophical literature during the last decades (Fricker 2007; Anderson 2012; Medina 2012; Pohlhaus 2017), only very recently was the attention of scholars attracted to the internal workings of the scientific community (Grasswick 2017; Perović 2017; Pronskikh 2018; Pla-Julián 2018). The account by Fricker (2007) originally identifies two types of epistemic injustice and considers them in relation to prejudice against social identities of certain discourse participants. As was extensively discussed above, contemporary big science, especially high-energy physics, exhibits a complex social structure. The 
community that constitutes it is stratified into subcommunities associated with certain epistemic and technical practices, which are unequal in their epistemic weight and value (Galison 1997). These communities develop various technical languages for communication, and some of them are functionally and linguistically subordinate to others which puts them in epistemically unequal positions (Pronskikh 2018).

In her original book, Fricker $(2007,158)$ suggested two types of epistemic injustice: testimonial injustice, which is due to systematic credibility discounting to people of certain disadvantaged or stigmatized social identities, and hermeneutical, which implies that members of socially marginalized groups lack resources to make meaning of their experience interpretable by the society. The remedies to both testimonial and hermeneutic injustices suggested by Fricker (2007) are respective virtues that individuals must exercise to counteract their prejudices. In Anderson (2012), the individual virtue-based remedies for epistemic injustices are challenged along two lines of argument. First, because cognitive biases are rooted deeply in the mind and have an automatic character, prejudicial hearers may discount the interlocutor's testimony because they perceive it incompetent or dishonest. Therefore, cognitive biases are difficult to control, although wellintentioned agents can train themselves to practice cognitive dissonance to discount their perceptions. Second, the credibility of the social groups can be discounted or favored not only on a transactional basis but also due to their belonging to a group (for example, certain ethnicity or using certain grammar). In the case, for example, of group favoritism or bias, there is no transactional injustice, on the contrary, in-group trust is vital in cases of division of labor (Anderson 2012, 170). Such cases cause, however, structural testimonial injustice and call for structural changes for their remedy. In her view, redesigning social institutions is unavoidable to mitigate structural epistemic injustice.

In the engineering context, the most relevant is seen as the concept of participatory injustice proposed by Hookway (2010) to clarify the forms of testimonial and hermeneutic injustices (Fricker 2007). Hookway (2010) pins down that "Participating is not just a matter of exchanging information: it involves asking questions, floating ideas, considering alternative possibilities, and so on." He concludes "epistemic injustice that is directed at someone's functioning as a participant in discussion, deliberation, and inquiry does not simply cause the victim to lose epistemic confidence more generally. Rather it questions the possession of capacities that are necessary for participation in these kinds of epistemic activities." (p. 6) Excluding engineers who, as we explained earlier, have most of the basic skills necessary for a scientist belonging to more epistemically significant discourses and practices, such as the collection and processing of experimental data, their discussion and presentation of the results of cognition outside, in our opinion is an example of epistemic injustice. In this regard, participatory injustice should be considered alongside other types of injustice, which is also structural in nature, i.e., requires institutional efforts, not just individual ones to transcend them.

Anatomizing the problem of institutional epistemic justice, Anderson (2012) points out that the epistemic segregation of the communities is just as unfair as ethnic or racial biases. However, collaborative learning and research can help overcome the bias of individuals and more privileged groups over less privileged ones. In the context of scientific and engineering communities, we believe that, in relation to scientific research and megascience in particular, collaborative learning and research can mean that representatives of separate communities (both detector and accelerator 
communities and, within these communities, research and engineering) should not only complete the same training courses, but also jointly discuss and contribute to all stages of research, from accelerated particles and facilities to data analysis and phenomenological theoretical calculations. Moreover, mobility between communities must be ensured, providing the opportunity and ability to move from engineers to scientists. This will help both to transcend the perception of boundary objects as delimiters between epistemic and nonepistemic communities and to fulfill the ethical requirement of epistemic equality, which is considered a condition of epistemic democracy.

\section{Conclusion}

This paper examines the community structure in high-energy physics, which for decades has been considered as including instrumentalists, experimenters, and theorists. In our view, the first two communities are more correctly regarded as accelerator and detector researchers, which can be divided into several groups, including engineers and other specializations. I seek to address the noted issue of a shift in the identity of the experimentalists and explain it through the convergence of the constructive nature of work of the experimentalist with the engineer as well as the advent of specialists of a narrower profile in the place of the classical experimentalist with epistemic division of labor. Under the conditions of a similar nature of labor against the background of narratively fixed perceptions about their purported scientific expertise and horizons, this could entail a certain crisis of the experimentalist's identity. We note that identities of the experimentalist and engineer began to blur and overlap, and their activity formulas nowadays almost coincide. The basis of the external distinction between engineers and nonengineers, as before, is the orientation of their constructive activities either toward the artificial, technical nature (among the former) or by natural phenomena (among the latter). At the same time, the engineering, constructing nature of labor turns out to be characteristic of both scientists and engineers, and the formal orientation of the activity toward artificial nature as a functional role, as a rule, serves as the basis for the refusal of engineering specialists to participate in experiments and analyze data. I argue that the exclusion of engineers and other nonscientist specializations in megascience from epistemically most valuable discourses and practices was considered by us in the framework of the concept of participatory epistemic injustice. I suggest an avenue of approach to overcome participatory injustice, such as joint projects for engineering and nonengineering specializations, in which they cast themselves in epistemically equipollent roles. 


\section{References}

Anderson, K., J. Boyett, et al. 2010. Understanding engineering work and identity: a cross-case analysis of engineers within six firms. Engineering Studies, 2 (3), 153-174.

Anderson, E. 2012. Epistemic justice as a virtue of social institutions, Social Epistemology: $A$ Journal of Knowledge, Culture and Policy, 26, 2 (2012): 163-173, doi: 10.1080/02691728.2011.652211

Boon, M. 2011. In Defense of Engineering Sciences. On the Epistemological Relations between Science and Technology. Techné. Research in Philosophy and Technology. 15: 49-73.

Collins, H.M. \& Evans, R.J. 2002. The third wave of science studies: Studies of expertise and experience. Social Studies of Sciences 32, no. 2: 235-296.

Fricker, M. 2007. Epistemic Injustice. Power and the Ethics of Knowing New York: Oxford University Press.

Galison, P. 1987. How Experiments End. Chicago and London: University of Chicago Press, 1987.

Galison, P. 1997. Image and Logic: A Material Culture of Microphysics. Chicago: University of Chicago Press, xxv+955 c.

Grasswick, H. 2017. Epistemic Injustice in Science In Ian James Kidd, José Medina, and Gaile Pohlhaus Jr. (eds.) Routledge Handbook of Epistemic Injustice pp. 13-26. Location: Routledge. doi:10.4324/9781315212043.

Hoddeson, L. et al. 2008. Fermilab: Physics, the Frontier, and Megascience. University of Chicago Press, Chicago, Illinois, 520 p.

Hookway, C. 2010. Some varieties of epistemic injustice: reflections on Fricker. Episteme, 7 no. 2: 151-163. doi:10.3366/E1742360010000882

Knorr-Cetina, K. 1999. Epistemic cultures: how the sciences make knowledge. Cambridge, Massachusetts: Harvard University Press, 1999. 352 p.

Latour, B. 1979. Laboratory Life: The Social Construction of Scientific Facts. Beverly Hills: Sage, $272 \mathrm{p}$.

Martin, J. D. 2017. "Prestige Asymmetry in American Physics: Aspirations, Applications, and the Purloined Letter Effect." Science in Context 30(4):475-506.

Medina, J. 2012. The Epistemology of Resistance: Gender and Racial Oppression, Epistemic Injustice, and Resistant Imaginations Oxford: Oxford University Press. 
Murphy M. et al. 2015. Designing the Identities of Engineers. In Engineering Education and Practice in Context, Vol. 2. Springer.

Perović, S. 2018. Egalitarian paradise or factory drudgery? organizing knowledge production in high energy physics (HEP) laboratories. Social Epistemology, 32 no. 4: 241-261, doi: 10.1080/02691728.2018.1466933.

Pickering, A. 1984. Constructing quarks: a sociological history of particle physics. Chicago, Illinois: University of Chicago Press.

Pla-Julián, I. and Jose-Luis, D. 2018, Gender Equality Perceptions of Future Engineers, Engineering Studies, DOI: 10.1080/19378629.2018.1530242

Pohlhaus Jr., G. ed. 2017. Varieties of epistemic injustice, In Routledge Handbook of Epistemic Injustice, pp. 13-26. Location: Routledge. doi:10.4324/9781315212043.

Pronskikh, V. 2018. Linguistic privilege and justice: what can we learn from STEM? Philosophical Papers, 47, no. 1: 71-92, DOI: 10.1080/05568641.2018.1429739.

Traweek, S. 1988. Beamtimes and Lifetimes: The World of High Energy Physics. Harvard University Press, Cambridge, MA, 1988. 206 p.

Wenger, E. 1998. Communities of Practice: Learning, Meaning and Identity. Cambridge, UK: Cambridge University Press. 